\title{
La salud durante el Cardenismo (1934-1940)
}

\author{
Martha Eugenia Rodríguez
}

Departamento de Historia y Filosofía de la Medicina, Facultad de Medicina, Universidad Nacional Autónoma de México, Ciudad de México, México

\section{Resumen}

El presente simposio, La salud durante el Cardenismo (1934-1940), se integra por cuatro estudios: Aspectos médico-sanitaristas en México, de Martha Eugenia Rodríguez; Campañas contra enfermedades, de Carlos Viesca Treviño; Los hospitales durante el Cardenismo, de Guillermo Fajardo Ortiz; y La medicina militar en México, de Antonio Moreno Guzmán. A través de ellos se da una visión integral del estado de salud y enfermedad durante la administración del General Lázaro Cárdenas del Río, primer gobierno sexenal del siglo XX. Se tratan varios aspectos. La política nacionalista del presidente llevó a un importante reparto de tierras a los campesinos. Su política educativa originó, entre otras cosas, la creación del Instituto Politécnico Nacional, que enmarcó dos escuelas de medicina, la Nacional de Medicina Homeopática y la Superior de Medicina Rural. Asimismo, se creó el servicio social para los pasantes de medicina de la Universidad Nacional Autónoma de México. Por otra parte, el General Cárdenas puso especial atención en la medicina preventiva y asistencial. Además de organizar campañas contra múltiples males, entre ellos viruela, tifo, tuberculosis, paludismo y enfermedades de transmisión sexual, se dio especial atención a la asistencia materno-infantil. Un problema urgente de atender fue el de la desnutrición, de manera que se prestó especial cuidado en la población infantil y campesina. Asimismo, para atender la morbilidad, en el periodo 1934-1940 hubo hospitales generales y de especialidad dependientes del gobierno, privados, militares y de beneficencia privada. El último estudio que se presenta hace referencia a las iniciativas de modernización sanitaria militar iniciadas por el General Cárdenas, que repercutieron en la salud de los militares y sus derechohabientes.

PALABRAS CLAVE: Periodo 1934-1940. Epidemias. Hospitales. Historia médico-militar. Prevención. Asistencia médica. Enfermedades. Campañas. Medicina militar.

\begin{abstract}
The present symposium, Health during the Cardenismo (1934-1940), consist of four studies: Medical sanitary aspects in Mexico by Martha Eugenia Rodríguez; Campaigns against diseases by Carlos Viesca Treviño; Hospitals during Cardenism by Guillermo Fajardo Ortiz; and Military medicine in Mexico by Antonio Moreno Guzmán. Through them is given an integral vision of the state of health and illness during the administration of General Lázaro Cárdenas del Río, the first sexennial presidential government of the twentieth century. Several aspects are discussed, among them, the President's nationalist policy which led to an important distribution of land to the peasants. His education policy originated, among other things, the creation of the National Polytechnic Institute that framed two medical schools, the National Homeopathic Medicine and the Superior of Rural Medicine. The social service for medical interns of the UNAM was created. On the other hand, General Cárdenas placed special emphasis on preventive and care medicine. In addition to organizing campaigns against multiple diseases, including pox, typhus, tuberculosis, malaria, and sexually transmitted diseases, special attention was given to maternal and child care. An urgent problem was that of malnutrition, so special care was taken in the child and peasant population. Likewise, in order to attend to morbidity, in the period 1934-1940, general and specialty hospitals were set up under government, private, military, and private charitable institutions. The last study that is presented refers to the military health modernization initiatives initiated by General Cárdenas, that had repercussions on the health of the military and its successors.
\end{abstract}

KEY WORDS: Period 1934-1940. Epidemics. Hospitals. Military medical history. Prevention. Medical assistance. Diseases. Campaigns. Military medicine.

\section{Correspondencia:}

Martha Eugenia Rodríguez

Brasil, 33

C.P. 06020, Ciudad de México, México

E-mail: martha.eugenia.rp@gmail.com
Fecha de recepción: 25-06-2017

Fecha de aceptación: 28-06-2017

DOI:10.24875/GMM.17003520
Gac Med Mex. 2017;153:608-625

Contents available at PubMed

www.gacetamedicademexico.com 


\title{
Aspectos médico-sanitaristas en México (1934-1940)
}

\author{
Martha Eugenia Rodríguez
}

Departamento de Historia y Filosofía de la Medicina, Facultad de Medicina, Universidad Nacional Autónoma de México, Ciudad de México, México

\section{Introducción}

El General Lázaro Cárdenas del Río se desempeñó como presidente de México del 1 de diciembre de 1934 al 30 de noviembre de 1940, siendo el primer gobierno sexenal del siglo XX. Cárdenas fue el último presidente que llegó al poder bajo la tutela de Plutarco Elías Calles, pero logró independizarse de él, dando fin al Maximato; es decir, al periodo comprendido entre 1928 y 1934, cuando México fue gobernado por Emilio Portes Gil, Pascual Ortiz Rubio y Abelardo L. Rodríguez, quienes fueron dominados por Elías Calles, presidente del país de 1924 a 1928 y conocido como «el jefe máximo de la revolución» (de ahí el término Maximato).

En el escenario internacional se da el ascenso del fascismo y el nazismo en Europa, así como la política del buen vecino instaurada por el presidente de los EE.UU., Franklin D. Roosevelt, hacia los países latinoamericanos'.

La política que emprendería el gobierno de Cárdenas se caracterizó por su nacionalismo, por alcanzar un equilibrio socioeconómico, por intensificar el reparto agrario y la necesidad de unificar y organizar a los trabajadores, de manera que expropió a mexicanos y extranjeros latifundios de los tiempos revolucionarios para distribuirlos en forma de ejidos a los campesinos, como fue el caso de la Comarca Lagunera en Durango y Coahuila, por una parte, y la zona henequenera en Yucatán, por citar solo unos ejemplos. Así, Cárdenas realizó el mayor reparto de tierras que se había hecho, creando más de 11,000 ejidos, que representaban el $10 \%$ del territorio nacional ${ }^{2}$ (Fig. 1).

El México de 1930 contaba con 16.5 millones de habitantes, de los cuales 11 millones residían en poblaciones rurales y 5.5 millones en localidades urbanas $^{3}$. Cabe mencionar que la política sanitaria del Cardenismo se enfocaría al crecimiento de la población, a fin de que se explotaran más los recursos naturales del país y se lograra un progreso definitivo (Tabla 1).

Por otra parte, con la Confederación de Trabajadores de México, erigida en 1936 bajo la dirección de Vicente Lombardo Toledano, militante convencido de la construcción de la sociedad socialista, se afianzó el movimiento obrero que simpatizaba con el gobierno. Cárdenas toleró las movilizaciones obreras y campesinas, la formación de sindicatos y las huelgas de empresas transportistas, mineras y petroleras ${ }^{4}$.

El pilar ideológico cardenista fue la defensa de la soberanía nacional ante la amenaza externa, y prueba de esa política fue la nacionalización de los ferrocarriles en 1937 y la expropiación petrolera el 18 de marzo del siguiente año, dado que el $100 \%$ de la industria estaba en manos extranjeras.

\section{Educación y cultura}

En su política cultural, Cárdenas implanta tres ideas rectoras: indigenismo, nacionalismo y justicia social. Así vemos, por ejemplo, que el arte se politiza, como se constata en el movimiento muralista mexicano realizado por José Clemente Orozco, Diego Rivera y David Alfaro Siqueiros ${ }^{5}$. Los tres pertenecieron al sindicato de pintores y escultores, intentando recuperar el arte de la pintura mural bajo el patrocinio del gobierno.

Cuestión aparte, en su informe al Congreso, el 1 de septiembre de 1935, el General Cárdenas participó la creación del Instituto Politécnico Nacional a fin de «impartir a las masas, particularmente a las proletarias, la capacitación técnica para que en la República

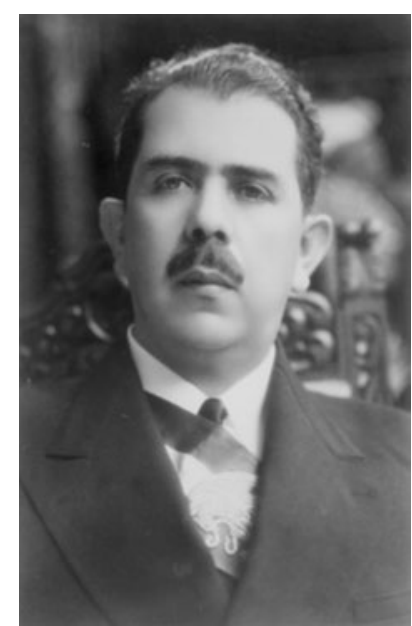

Figura 1. El General Lázaro Cárdenas del Río, titular del primer gobierno sexenal de México en el siglo XX. 
Tabla 1. INEGI

\begin{tabular}{|c|c|c|c|c|}
\hline Año & País & Habitantes & Capital & Habitantes \\
\hline 1921 & Estados Unidos Mexicanos & 14334780 & Distrito Federal & 906063 \\
\hline 1930 & Estados Unidos Mexicanos & 16552722 & Distrito Federal & 1229576 \\
\hline 1940 & Estados Unidos Mexicanos & 19653552 & Distrito Federal & 1757530 \\
\hline
\end{tabular}

Mexicana cada individuo sea una unidad social más apta para el ejercicio colectivo.... ${ }^{6}$. El Politécnico contó con varias escuelas, entre ellas la Nacional de Medicina Homeopática fundada en 1889, pero fusionada al Politécnico por medio de un decreto. De igual manera, el Instituto citado enmarcó la Escuela Nacional de Ciencias Biológicas, que amparó en 1938 la creación de la Escuela Superior de Medicina Rural, denominada con este adjetivo hasta $1965^{7}$. Bajo ese contexto, el presidente Cárdenas se afanaba por extender los servicios de salud a la población vulnerable, particularmente a la campesina. Con la orientación social que caracterizaba a esta última Escuela, que impartía una formación técnica en aspectos de salubridad general, el gobierno mexicano pretendía resolver los problemas médicos del día a día.

\section{Servicio Social}

En concordancia con la política nacional cardenista, en 1936 se creó el Servicio Social para los pasantes de la carrera de medicina de la Universidad Nacional. Su instauración significaba una manera de resolver problemáticas sociales, fundamentalmente en los medios rurales; de retribuir a la sociedad y beneficiar a la población más necesitada, por lo que se estableció un acuerdo entre el Departamento de Salubridad Pública, institución rectora de la medicina, y la Universidad Nacional Autónoma de México (UNAM), dirigidas en ese momento por el Dr. José Siurob Ramírez y el Lic. Luis Chico Goerne, respectivamente. Para ello, el Dr. Gustavo Baz Prada, director de la Escuela Nacional de Medicina, consideró al pasante como un promotor para trabajar en las comunidades rurales del país, señalando en el discurso que dirigió a la primera generación de pasantes que ejercerían «su ministerio entre gentes primitivas y rudísimas, hijos incultos de la sierra, del bosque y del páramo que no tienen la más leve noticia de lo que es el moderno arte de curar»». Así, el Dr. Baz señaló que el servicio sería un requisito para obtener el título de médico y consistiría en una práctica en el medio rural durante 5 meses $^{9}$. Sin embargo, los trámites y traslados reducían el trabajo efectivo a 3.5 meses, razón por la que posteriormente el servicio fue ampliado a un año. El Lic. Roberto García Formentí fue el primer jefe de la oficina de distribución de médicos.

\section{Exilio español}

Con la Guerra Civil Española y la derrota republicana en 1939, se estima que al instaurarse la dictadura de Francisco Franco hubo más de 6000 refugiados registrados en México $^{10}$. En ese contexto, un nutrido grupo de intelectuales tuvo cabida en nuestra máxima casa de estudios. Llegaron eminentes figuras que contribuyeron al desarrollo del derecho, la filosofía, la biología, la química y la medicina mexicana, entre ellos Isaac Costero Tudanca (1903-1979), quien fuera miembro de la Escuela de Histología Española presidida por Santiago Ramón y Cajal. Los doctores Tomás G. Perrín e Ignacio Chávez instaron a Costero Tudanca para que continuara sus trabajos docentes y de investigación anatomopatológica en el Hospital General, en la UNAM y en el Instituto Nacional de Cardiología, entre otras instituciones. De hecho, fue Costero quien inauguró una segunda época de la investigación anatomopatológica en México, ya que la iniciada por Rafael Lavista y Manuel Toussaint a finales del siglo XIX, se vio interrumpida por el movimiento revolucionario de 1910. Durante el desempeño de sus actividades, el destacado médico español afirmaba que «al descubrimiento científico debe seguir su interpretación filosófica», logrando establecer bases metodológicas para la investigación ${ }^{11}$. Un hecho a subrayar, y en reconocimiento a la trayectoria de Costero Tudanca, es que en 1968 fue electo para ocupar la presidencia de la Academia Nacional de Medicina de México.

\section{El plan sexenal y la medicina}

El plan de gobierno para el periodo 1934-1940 señaló varios puntos a favor de la medicina preventiva y asistencial, que serían analizados y atendidos a 
través de la institución rectora de la medicina, el Departamento de Salubridad Pública. Durante el sexenio, los titulares de dicha institución fueron Abraham Ayala González (1934-1935), José Siurob Ramírez (1935-1938), Leonides Andrew Almazán (1938-1939) y nuevamente Siurob (1939-1940).

Del presupuesto federal reservó un monto para la salubridad, que se incrementaría gradualmente a fin de ampliar los servicios sanitarios, entre ellos los de agua y atarjeas. Respecto al presupuesto en salud, en 1935 fue de 7.4 millones de pesos, lo que significaba el 3.4\% del producto interior bruto (PIB). En 1939 fue de 16.5 millones de pesos; es decir, el $5.5 \%$ del PIB. Interesaba reservar ese incremento a la salud de campesinos y obreros, dado que representaban importantes fuentes de energía para el país ${ }^{12}$.

El presidente Cárdenas se comprometió a hacer frente a las enfermedades reinantes, particularmente la tifoidea, el sarampión, la escarlatina, la tos ferina, la difteria, el paludismo, la fiebre amarilla y el tifo; a combatir las epidemias y endemias, entre ellas la tuberculosis, la lepra, la sífilis, el paludismo, la oncocercosis y la viruela; a formar personal técnico sanitario que cubriera toda la geografía nacional; a prestar atención en la alimentación popular y dar continuidad al trabajo descentralizado por medio de la Coordinación y Cooperación de los Servicios Sanitarios Federales y Locales de la República.

Un punto importante del gobierno cardenista fue la protección a la infancia y la lucha contra la mortalidad infantil. El Dr. José Siurob, titular del Departamento de Salubridad Pública decía: "La protección a la infancia no es un asunto sentimental ni de caridad, sino que es un serio problema social que implica la creación de una población sana y fuerte», que constituyera la unidad de la nación. Así, la protección a los niños empezó desde los exámenes prematrimoniales, la vigilancia del embarazo, el parto, la defensa de la lactancia y el crecimiento del infante ${ }^{13}$.

Al iniciar el gobierno cardenista, las estadísticas de mortalidad anual ${ }^{14}$ en 1935 fueron las siguientes:

- 100,000 de población infantil.

- 83,000 del aparato digestivo.

- 62,000 del aparato respiratorio.

- 25,000 de paludismo.

- 12,000 por tuberculosis.

- 7000-8000 por viruela.

- 6000 por disentería.

Para tales enfermedades se intensificaron las campañas preventivas y asistenciales, cobrando importancia la enfermera visitadora, que tenía a su cargo la impartición de la educación higiénica. Tras hablar con los enfermos, en lenguaje claro, les daba una somera «explicación científica» sobre la transmisión de las enfermedades. Las enfermeras sanitarias debían tener conocimiento sobre las enfermedades de notificación obligatoria, la etiología, la fuente de infección, la manera de transmisión, el periodo de incubación, el periodo de infección, las pautas de diagnóstico, las medidas preventivas, las inmunizaciones y la investigación de portadores. De igual manera, la enfermera podía intervenir en los aspectos sociales, como la mejora de la alimentación y la vivienda ${ }^{15}$. En fin, el papel de la enfermera sanitaria fue de gran relevancia (Fig. 2).

Cuestión aparte, para combatir la viruela se emitió un reglamento el 16 de agosto de 1935 señalando la obligatoriedad de la vacuna. La instrucción también contempló que los jefes de establecimientos públicos 0 privados, como cárceles, corporaciones militares y de policía, tenían el compromiso de vacunar a sus integrantes. La Iglesia solo realizaría confirmaciones cuando se presentara la cartilla de vacunación del infante.

Asimismo, el Departamento de Salubridad Pública fomentó la medicina social y prestó atención en los problemas que se vivían día a día, como el alcoholismo y la prostitución. De igual manera, vinculó la pobreza con la enfermedad, analizando el escenario en que vivía el enfermo. De manera que, entre este Departamento y los Servicios Sanitarios Federales, desarrollaron tareas conjuntas: primero, tratar de averiguar los factores que generaban las enfermedades, como las costumbres de la población, identificando en qué clima y vivienda habitaban; y en segundo término, hacer inspecciones de alimentos y bebidas, emprender obras de ingeniería sanitaria (como letrinas, por ejemplo), eliminar mosquitos y elaborar estadísticas a fin de conocer el número de afectados por una determinada enfermedad.

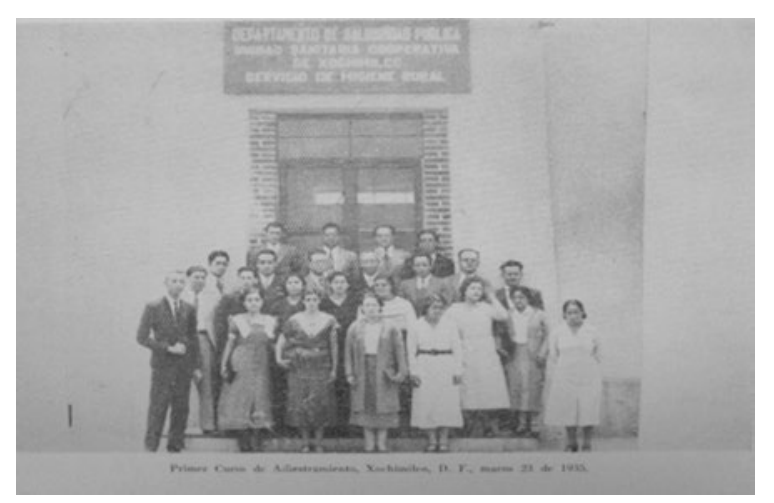

Figura 2. Impartición del primer curso de adiestramiento del Servicio de Higiene Rural, 1935 (en: J. Álvarez Amézquita, Historia de la Salubridad y de la Asistencia en México, 1960). 


\section{Comentarios}

La directriz cardenista, que pretendía alcanzar la equidad entre la población mexicana, orientó su política sanitaria hacia la prevención de enfermedades, fundamentalmente las infantiles y en segundo término vio por el tratamiento asistencial, particularmente a obreros y campesinos. De igual manera, se interesó por las campañas sociales, como la antialcohólica, para crear conciencia sobre la población mexicana ${ }^{16}$.

La erradicación de las enfermedades fue el tema central de la salud pública, que significaba no sólo un instrumento para optimizar la economía sino también para la mejora a nivel individual. Por tanto, a través de campañas y brigadas sanitarias móviles se dio prioridad al cuidado de enfermedades de los aparatos digestivo y respiratorio, así como las causas que las creaban, por lo que el gobierno cardenista prestó atención a diversos asuntos, como la mala calidad de los alimentos y del agua, además de emprender obras de ingeniería sanitaria.

En opinión de Álvarez Amézquita17, al término del sexenio cardenista hubo un progreso significativo en cuanto a materia sanitaria se refiere. Este logro se debió al trabajo conjunto de diversos organismos, el Departamento de Salubridad Pública como la institución que regía la medicina, pero también al Instituto de Higiene, la Escuela de Salubridad, el Instituto de Salubridad y Enfermedades Tropicales, la Secretaría de Asistencia Pública y los centros de higiene rural distribuidos por todo el territorio nacional. Jugaron un papel importante los médicos, pero también las enfermeras sanitarias, las trabajadoras sociales, ingenieros y técnicos. Todos sumaron esfuerzos para trabajar por un desarrollo nacional integrado.

Respecto a lo político, si bien es cierto que en su momento la tendencia socialista tuvo una amplia aceptación entre la población, el radicalismo de Cárdenas fragmentó al país. En 1939 se conformó el Partido Acción Nacional (PAN), bajo la dirección del abogado Manuel Gómez Morín (18) y se multiplicaron no sólo las oposiciones internas sino también las de los intereses extranjeros afectados por las expropiaciones, de manera que en las siguientes administraciones la vida política cambió su directriz. 


\title{
Campañas contra enfermedades durante el Cardenismo
}

\author{
Carlos Viesca-Treviño \\ Departamento de Historia y Filosofía de la Medicina, Facultad de Medicina, Universidad Nacional Autónoma de México, Ciudad de México, México
}

\section{Introducción}

El período presidencial de Lázaro Cárdenas, 1934-1940, marcó el inicio de una tendencia a la estabilidad en el país y la apertura de posibilidades de desarrollo de los ideales e idearios de la Revolución Mexicana. Dos aspectos prioritarios de su política y su gobierno fueron la educación y la salud, rubros en los que se llevaron a cabo acciones que fincaron los rumbos que tomarían y seguirían ambas en las décadas siguientes.

\section{El plan sexenal de 1934 y su conversión en acciones}

Elaborado entre 1933 y 1934, este primer plan sexenal establecía un compromiso por parte del Ejecutivo para establecer lineamientos políticos definidos y llevar a cabo una serie de acciones conducentes a la solución de problemas concretos. En el ámbito de la salud quedó claramente definida la necesidad de restablecer las condiciones higiénicas perdidas durante los años de la lucha armada, junto con la recuperación del sistema hospitalario, actualizándolo y modernizándolo, pero sobre todo de llevar a cabo el estudio objetivo de los problemas a atacar, desarrollando la captación sistemática de los datos estadísticos y llevando a cabo acciones concertadas para combatir las enfermedades transmisibles, así como los focos de endemias y epidemias. Es indiscutible que se reconoció la importancia y la urgencia de impulsar políticas de salud que respondieran a las necesidades ya conocidas y a las nuevamente detectadas ${ }^{19}$.

En su primer informe de Gobierno, el 1 de septiembre de 1935, reafirmaba el camino abierto a una política pública dirigida a «intensificar las actividades de higienización y propaganda sanitaria» tanto en las ciudades como en las áreas rurales ${ }^{20}$. A 10 meses de su toma de posesión, Cárdenas afirmaba que se habían llevado a cabo «importantes campañas de vacunación en todos los sectores del país", que se había creado un Departamento de Higiene Industrial a fin de «proteger la salud de los trabajadores del campo y de la ciudad", la instalación de dispensarios antituberculosos, la intensificación de la lucha contra las enfermedades venéreas, el combate contra la oncocercosis, el mal del pinto y la lepra. Se hacía hincapié en la urgencia de dotar de aguas potables a la población, dada la alta incidencia de enfermedades gastrointestinales y de muertes asociadas a ellas ${ }^{21}$.

\section{Algunos datos reveladores: de 1934 a 1940 las estadísticas revelan cambios}

En 1930, la población del país era de 16,552,782 habitantes y para 1940 había aumentado a 19,650,000 habitantes, 1,800,000 de ellos en la Ciudad de México. La expectativa de vida al nacer, que era de 33 años, pasó a ser de 41.5 años; la tasa de defunciones pasó de 26.6 a 22.8 por 1000 habitantes, y la mortalidad de niños menores de un año pasó de 250 a 24.5 por 1000 nacidos vivos. La tasa de natalidad se mantuvo estable en 44.6 por 1000 habitantes $^{22,23}$.

\section{Una visión epidemiológica en 1934}

En 1934, Miguel Bustamante, que era entonces subdirector de la flamante Dirección de Servicios Coordinados, presentaba una visión de conjunto de las condiciones epidemiológicas del país. Su análisis se enfocó primordialmente a las causas de muerte, señalando que, en términos generales, la mayor parte de los Estados de la República tenían tasas de mortalidad al año que iban entre 31 y 40 por 1000 habitantes, con un coeficiente general que nunca fue menor de 26 por 1000. Para 1934 eran las neumonías y las enfermedades agudas respiratorias la primera causa de muerte, seguidas por diarreas y enteritis, con las fiebres tifoidea y paratifoidea en segundo lugar, el paludismo en tercero, seguido del sarampión y la tosferina, y con la viruela en el sexto. Es de hacer notar que el $42.6 \%$ de las defunciones se debían a enfermedades de origen infeccioso y parasitario, y que esto no cambió durante el sexenio, aunque comenzó a decrecer paulatinamente después de 1940. La comparación con cifras procedentes de otros países llevaba a considerar al nuestro como deficiente en sus condiciones higiénicas e invocaba la necesidad imperativa de organizar servicios 
sanitarios orientados a establecer los tratamientos y las medidas de prevención necesarios ${ }^{24}$.

La doctrina central que se invocaba para impulsar los cambios en las políticas sanitarias y de atención médica era la de la medicina social, sostenida por Alfonso Angelini desde 1933, la cual sería la bandera principal de las políticas de salud del Cardenismo ${ }^{25}$.

\section{La viruela}

La Administración anterior a Cárdenas fue testigo de los esfuerzos de los doctores Gastón Melo, Manuel Gea González y Manuel Madrazo para promover la vacunación contra la viruela, y los resultados no tardaron en dejarse sentir: de 17,405 defunciones en 1930 se había llegado a 6,094 en 1933, cifra que subió a 9,430 en 1934, en razón del brote epidémico que afectó a los poblados de la sierra de Oaxaca y gran parte de Chiapas. Esto motivó un interés renovado en la vacunación. Para el año siguiente, 1935, fueron 6,000,000 las dosis aplicadas y paralelamente se hace público un nuevo reglamento de vacunación y revacunación. En 1936 se presentó un brote en Tlapacoya, Veracruz, el cual fue controlado en 3 meses y seguido de intensas campañas en Oaxaca, en particular en Pochutla, Candelaria, Tuxtepec, Putla, Silacayoapan y Ocotlán ${ }^{26}$. El resultado final fue que durante el sexenio del gobierno del General Cárdenas la mortalidad por viruela fue disminuyendo paulatinamente, de 5205 casos en 1935 a 1341 en 1940, a lo que contribuyó notablemente la producción esmerada de la vacuna por parte del Instituto de Higiene ${ }^{27}$. Habría un nuevo brote en 1941 que se continuó hasta 1943, es decir, ya terminado el mandato de Cárdenas, pero debe señalarse que fue combatido de manera enérgica y oportuna, y que se logró una continuidad

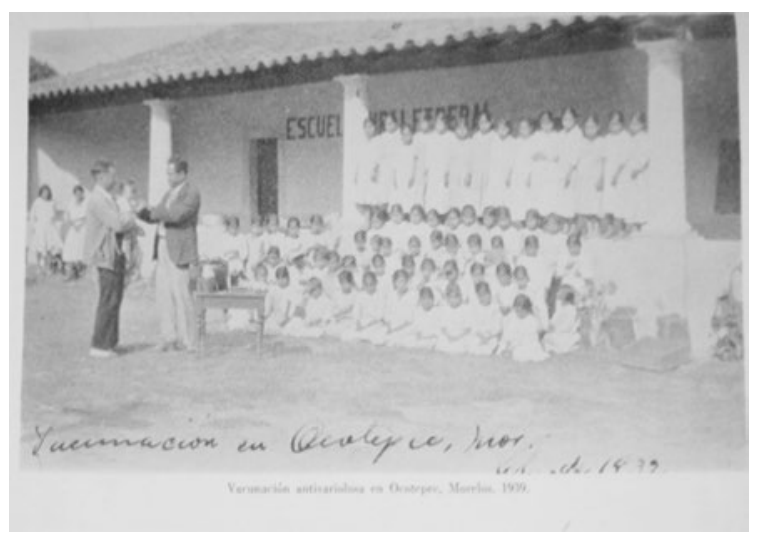

Figura 3. Campaña antivariolosa. Departamento de Salubridad Pública, 1934 (en: J. Álvarez Amézquita. Historia de la Salubridad y de la Asistencia en México, 1960). de acciones más allá de los cambios de gobierno, lo que condujo a la erradicación de la enfermedad en $1952^{28}$ (Fig. 3).

\section{El tifo}

Si bien no hubo epidemias importantes de tifo durante el período aquí referido, esta enfermedad no dejó de ser una preocupación constante, en particular debido a las epidemias importantes y a la multitud de brotes que se presentaron durante los años de la Revolución y en los diversos levantamientos militares de los años subsecuentes. Un último brote de considerable magnitud se dio en la capital en 1929, y cuarteles, casas de niños expósitos, reclusorios y hospitales no dejaban de documentar casos a pesar de las medidas de despiojamiento, que cada vez se hacían más constantes. Tras haber aislado en la década anterior la rickettsia causal del tifo mexicano, mediante sus trabajos personales y los hechos en colaboración con Hans Zinsser y Hermann Mooser, en 1937 Maximiliano Ruiz Castañeda inaugura el laboratorio de estudios sobre el tifo en el Hospital General, en el cual desarrollaría finalmente una vacuna antitífica efectiva, la cual se empleó sistemáticamente en las campañas implementadas para tal efecto y sería regalada por el Gobierno Mexicano para ser utilizada en Polonia en la terrible epidemia que la azotó a raíz de la invasión alemana ${ }^{29}$.

\section{Tuberculosis}

Unos meses antes del acceso de Cárdenas a la presidencia, en febrero de 1934, Manuel Gea González había logrado echar a andar la Campaña Antituberculosa, retomando la idea de Liceaga de organizar sistemas para la detección temprana de la enfermedad, instaurar tratamiento de inmediato y promover la construcción de un sanatorio especializado en el manejo de estos enfermos. Pronto se inició la construcción de este, que sería el Hospital de Huipulco, el cual fue inaugurado el 31 de diciembre de 1935. Es también en 1935 cuando se comienza a preparar vacuna antituberculosa con el bacilo de Calmette-Guerin en el Instituto de Higiene, y en 1938 se estableció la utilización de las reacciones de von Pirquet y Mantoux y el DPP (derivado proteico purificado). Para 1940 se calculaban de 13 a 14,000 muertes al año por tuberculosis ${ }^{30}$.

La lepra y el mal del pinto fueron por igual objeto de atención. Una campaña nacional contra la lepra 
llevó a la construcción del Hospital Pascua, creado a instancias de Fernando Latapí, el cual fue inaugurado el 2 de enero de 1937. El estudio del segundo llevó a la identificación del agente causal por Salvador González Herrejón y a que en su honor fuera denominado Treponema herrejoni, en tanto que, en 1939, Eduardo Aguirre Pequeño, en Monterrey, se autoinocula suero y contrae la enfermedad, comprobando así la naturaleza contagiosa de esta y, sobre todo, la transmisión del agente causal.

\section{Paludismo}

El paludismo seguía siendo un grave problema de salud pública, de modo que en 1936 se declaró de interés especial la campaña en su contra. Se pone especial atención en precisar las zonas endémicas, identificándose en 1937 la costa en la colindancia entre Tamaulipas y Veracruz, y a raíz de haberse declarado oficialmente en 1938 la lucha contra el paludismo como de utilidad pública, en 1939, el 24 de marzo, se emite un timbre postal y se establece que todos los recursos generados por este se apliquen a la campaña de erradicación. Empero, esta campaña inicial, continuación de las que antes había llevado a cabo Liceaga a principios del siglo, prosiguió hasta 1956, cuando fueron establecidos nuevos lineamientos.

La fundación del Instituto de Enfermedades Tropicales concentró los esfuerzos para el estudio de este tipo de problemas y constituyó un hito en la evolución de la medicina mexicana. Su primer director fue Manuel Martínez Báez ${ }^{31}$.

\section{Oncocercosis}

La oncocercosis no fue tomada en consideración hasta los trabajos de Lacombe en la década de 1920. Para 1934 había de 22,000 a 25,000 afectados en Chiapas y entre 5000 y 8000 en Oaxaca. El 13 de abril de 1935 se publica el reglamento que establece la campaña contra ella, y en 1940 se llevan a cabo acciones extraordinarias al respecto en ambos Estados.

\section{Cisticercosis}

Aunque la teniasis era bien conocida, la presencia de cisticercos en el cerebro es detectada apenas en 1938, cuando Leopoldo Salazar Viniegra diagnostica y reporta el primer caso de cisticercosis cerebral y José Rojo de la Vega opera un caso de cisticercosis racemosa cerebral en el Hospital Juárez. Un año después, Clemente Robles muestra estadísticamente la importancia de la neurocisticercosis en el Hospital General.

Otras parasitosis salen a la luz, como la enfermedad de Chagas en humanos, detectada en 1939 por Mazzotti en Oaxaca, siendo reportado un caso en Yucatán un año después.

\section{La asistencia materno-infantil}

La preocupación por la salud de las mujeres durante el embarazo, el parto y el puerperio, y de los recién nacidos, que tuviera un desarrollo importante en la década anterior, continuó siendo prioritaria.

Un problema urgente de atender era el de la desnutrición. Ya se habían llevado a cabo acciones, como la gota de leche, encaminadas a mejorar y garantizar el acceso de los lactantes al suministro de leche, al tiempo en que se diseñaban esquemas adecuados de destete. Los trabajos de Manuel Cárdenas de la Vega, y luego de Federico Gómez y Rigoberto Aguilar Pico, en la Casa de Cuna y el Hospital Dolores Sáenz de Lavie, fueron fundamentales, y ya en 1934 Gabriel Araujo, en su tesis recepcional, proponía utilizar harina de garbanzo en la dieta. Vendría a continuación una serie de estudios clínicos que conducirían a Federico Gómez a definir con precisión los grados de desnutrición en la escala que sigue en uso hasta la actualidad $^{32}$.

La pelagra fue una enfermedad que, siempre presente, había sido poco estudiada. Ahora, en Yucatán, Álvaro Carrillo Gil, entre 1932 y 1936, estudia y publica la xeroftalmia y la pelagra (culebrilla), siendo pionero en el estudio clínico de la desnutrición.

Motivo fundamental de desarrollo en salud fue la atención materno-infantil, encaminada a disminuir la morbilidad y la mortalidad durante el embarazo y el parto en el caso de la madre, a la que se suma la de los primeros meses de vida del niño. En 1936 son inauguradas las primeras unidades de este tipo de atención en Xalapa, Tuxpan y Coatzacoalcos, Veracruz, teniendo como meta la atención integral de la familia en la comunidad con programas de saneamiento ambiental, vacunación y asistencia médica. En 1937 inicia sus labores un nuevo Departamento Autónomo de Asistencia Social e Infantil con desayunos para niños, a cargo de Isidro Espinosa de los Reyes y Salvador Zubirán, y ese mismo año Matilde Rodríguez Cabo publica El control de la natalidad señalando los puntos más importantes de la eugenesia y del control de las enfermedades de transmisión sexual, 
siendo la sífilis congénita y las oftalmías purulentas dos causas de grandes males. Para el manejo de la segunda se hizo obligatorio el uso de gotas a base de plata (método de Credé). Todo esto culminó en 1939 con el establecimiento de la Campaña Nacional en pro de la madre y el niño. Antes, en 1937, se determina dar prioridad a la construcción de hospitales para niños, que culminará con la fundación del Hospital Infantil. Para 1940, las muertes maternas habían disminuido a 56.5 por 10,000 .

\section{Enfermedades de transmisión sexual}

Elíseo Ramírez promovió una campaña antivenérea tendiendo a abolir la prostitución reglamentada, lo que llevó a crear en 1936 la Sección Técnica de la Campaña contra las enfermedades venéreas. El aspecto educativo fue cubierto por Alfonso Pruneda en su libro El médico y la educación sexual, y en 1939 se establece la Liga Nacional contra el Peligro Venéreo, fundándose nuevos dispensarios con ese preciso fin, todo lo cual culmina en 1940 con la Campaña contra las enfermedades venéreas, incluyendo sífilis, chancro blando, blenorragia, granuloma venéreo y enfermedad de Nicolás y Favre (Fig. 4).

\section{Toxicomanías}

Las toxicomanías son otro rubro que cobró relevancia durante este período. El alcoholismo ocupó el papel más relevante, pero no se dejaron de identificar

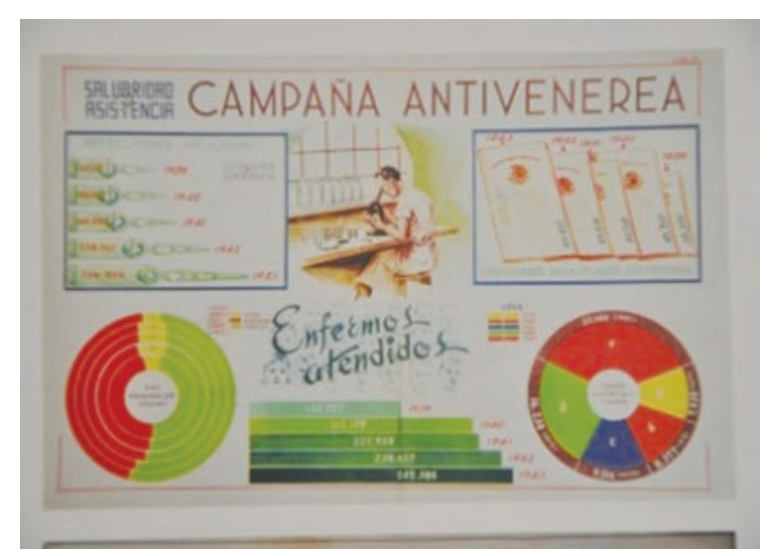

Figura 4. Campaña contra las enfermedades de transmisión sexual. Departamento de Salubridad Pública.

y buscar soluciones médicas a otras toxicomanías, en especial la dependencia a la mariguana y a los opiáceos. El 11 de noviembre de 1935, la Secretaría de Asistencia Pública establece un subcomité femenino de cooperación antialcohólica, y es por estas fechas que se funda el Pabellón de toxicómanos en La Castañeda, bajo el rubro, promulgado por Leopoldo Salazar Viniegra en 1939, de que los drogadictos son enfermos, no criminales. Es en este mismo sentido que se establece que para evitar el tráfico de drogas se haga un registro de adictos, se registre también el tipo de droga y las dosis que consumen, y de acuerdo con estos datos, con un control estricto y bajo receta oficial, se les entregue periódicamente a cada uno de ellos la cantidad de droga así especificada en la farmacia central de la Secretaría de Asistencia. 


\title{
Los hospitales durante el Cardenismo (1934-1940)
}

\author{
Guillermo Fajardo-Ortiz
}

Subdivisión de Educación Continua, División de Estudios de Posgrado, Facultad de Medicina, Universidad Nacional Autónoma de México, Ciudad de México, México

\section{Los marcos políticos}

Esta semblanza hace un recuento breve de hechos importantes en los hospitales del sexenio (1934-1940); mucha agua ha corrido bajo el puente desde entonces. En el arranque de ese sexenio ya no sonaban los disparos, las autoridades públicas asumieron que existían serios e importantes problemas de diversa índole, y tuvieron como objetivo y obligación la paz social y la vida jurídica. El país reflejó signos de cambio, son tiempos posrevolucionarios. El escritor Jean Meyer expresó: «después de 24 años de violencia casi ininterrumpida, entre 1934-1940 ocurre el último capítulo de la etapa revolucionaria, con la revolución pacífica realizada por el presidente Lázaro Cárdenas, el primero en durar seis años en el poder »33. En el período en estudio, la hoy Ciudad de México era un lugar remoto para la «provincia». En los Estados y territorios pocas carreteras eran asfaltadas, había caminos perdidos, los ferrocarriles eran lentos, las líneas telefónicas eran inciertas, el tiempo parecía haberse congelado. En esos años se crearon nuevos caminos médico-sanitarios, los que fueron consecuencia de la reforma agraria, la nacionalización de los ferrocarriles, el asilo político a los exiliados españoles, la expropiación petrolera y los avances técnico-científicos. Persistieron hospitales originados en la época colonial, creados en la etapa porfirista y estructurados en los tiempos armados de la Revolución Mexicana. En los hospitales de 1934 a 1940 se encuentran filosofía, historia, medicina, educación e innovación, no solo de ese sexenio, sino del futuro. Hay una gran variedad de información médico-sanitaria, social, política, económica y ética.

Hay que tener en cuenta la escasez de datos fiables respecto al número de hospitales y camas en los mismos en los ámbitos nacional, estatal, territorial y local, pero probablemente había menos de 20,000 camas de hospital. México, al terminar el sexenio en estudio, tenía 20 millones de habitantes, con lo cual había quizás menos de una cama de hospital por mil habitantes, y no existía la certificación de hospitales.

Las condiciones estructurales socioeconómicas, como la dispersión de la población en el territorio nacional, combinada con la escasez de recursos

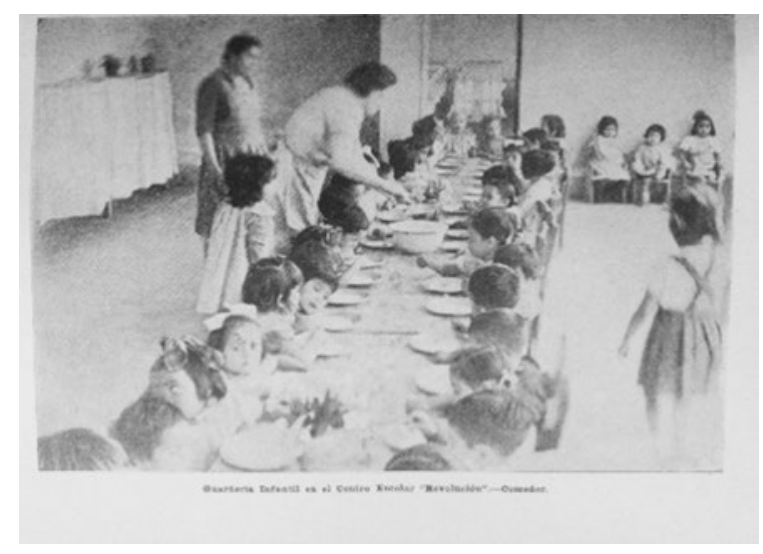

Figura 5. Guardería infantil (en: J. Álvarez Amézquita. Historia de la salubridad y de la asistencia en México, 1960).

económicos, condujo a una concentración de los hospitales en las ciudades (Fig. 5).

\section{Los pies y las cabezas de los hospitales}

Los hospitales, desde el punto de vista técnico y administrativo, eran sencillos, y el equipo y el instrumental eran simples. Los medicamentos muchas veces eran fórmulas magistrales. La atención médica estaba en manos del sexo masculino, y prácticamente no había enfermeras tituladas.

Los hospitales en funcionamiento databan de la etapa colonial, de los tiempos porfiristas y de los tiempos armados de la Revolución Mexicana. Entre los primeros estaban el Hospital de Jesús en la Ciudad de México y el Hospital Belén en Guadalajara. Posteriormente se crearon el hoy Hospital General Dr. Eduardo Liceaga, en la Ciudad de México, y el Hospital General O’Horan, en Mérida, Yucatán. De los tiempos revolucionarios había instalaciones nosocomiales de la Cruz Roja, la Cruz Verde y la Cruz Blanca; en su caso eran establecimientos de "sangre».

En relación a la rama de la medicina, se contaba con hospitales generales y especializados. Entre estos últimos se encontraban manicomios, establecimientos oftalmológicos, maternidades, hospitales para urgencias, leprocomios, hospitales para tuberculosos y hospitales para atender a personas con enfermedades infectocontagiosas. En los hospitales generales se atendían básicamente las enfermedades 
llamadas «agudas», partos y accidentes. No se conocían los niveles de atención médica y la Clasificación Internacional de Enfermedades era prácticamente desconocida.

Las construcciones hospitalarias, en cuanto a diseños médico-arquitectónicos, correspondían básicamente con el tipo clerical, que databa de la época colonial, y el tipo pabellones, de los tiempos del porfirismo. Se construyeron los hospitales verticales.

En cuanto a dependencia se refiere, había cuatro variedades: gubernamentales, de iniciativa privada, militares y de beneficencia privada. Entre los gubernamentales había federales, estatales y municipales; en general eran destinados para la población en pobreza. En el rubro de iniciativa privada había lucrativos y los que pertenecían a los trabajadores. Un ejemplo de los primeros fue la Clínica Neuro-psiquiátrica Dr. Manuel Falcón, en la Ciudad de México; entre los segundos estaban los que eran para mineros, azucareros, cañeros, cinematograficistas, petroleros, ferrocarrileros y para algunos grupos de burócratas, entre los que se encontraba el Hospital de la Secretaría de Hacienda y Crédito Público. Los hospitales militares eran para elementos de la secretaría de guerra y marina. Los de beneficencia privada pertenecían a las «colonias» española, francesa, americana y británica, y eran básicamente para sus afiliados. En este grupo también estaban los de la Cruz Roja. En esos años, como ahora, la atención médica estaba fragmentada y segmentada, y había inequidad.

\section{Atención a los campesinos}

En el aspecto gubernamental, para el medio rural las autoridades federales establecieron en 1936 un programa de salud dedicado a grupos agrarios, conocido como "servicios médicos rurales cooperativos. Eran sostenidos con cuotas simbólicas de la población campesina y aportaciones del gobierno federal. Con este modelo se le otorgó dignidad al lugareño. Los servicios fueron una importante expresión, no solo en cuanto a atender problemas de salud, sino que además se orientaron ayudar y mejorar la economía agrícola. La creación de dichos servicios fue un acontecimiento extraordinario. Reapareció el indígena como protagonista importante en la atención médica; hombre vestido de manta, usaba huaraches y sombrero de palma. Los servicios, además de hospitales, tuvieron centros de higiene rural y unidades sanitarias. En estos últimos se atendían no solo problemas clínicos, sino también necesidades de salud pública. Había instalaciones referentes a agua potable, drenaje y fosas sépticas. Los inmuebles de los servicios eran de bajo costo, estaban asentados en tierra, arena y lodo, con frecuencia no contaban con mobiliario y equipo suficiente, había escasez de medicamentos, y aun el alcohol, que se consideraba indispensable, faltaba. En cambio, había un inmenso deseo de ayudar y de servir las 24 horas del día a los campesinos. Este esfuerzo fue producto de estudios y trabajo de sociólogos, sanitaristas y funcionarios públicos, personas apasionadas, optimistas y soñadoras. En documentos del archivo histórico de la Secretaría de Salubridad y Asistencia se expresa: «las autoridades federales ampliaron la cobertura clínica y sanitaria, crearon los servicios médicos-sanitarios colectivos, que dependían de la oficina central de higiene rural». En otra sección, los documentos escritos expresan: «en 1936 había 36 unidades en todo México» ${ }^{34}$. Dos hospitales importantes de dichos servicios fueron el de Ciudad Anáhuac, en el Estado de Nuevo León, y el de Mérida, en el Estado de Yucatán, que fueron unidades de concentración. Los servicios obedecieron de alguna forma a lo formulado en la Sección de Salubridad Pública del plan sexenal formulado por el gobierno cardenista, entrante en $1934^{35}$.

\section{Iconos hospitalarios}

En los 6 años en estudio, el gobierno federal conformó hospitales públicos de vanguardia, de los que se mencionan tres: el Sanatorio de Tuberculosis y el Instituto de Salubridad y Enfermedades Tropicales en la Ciudad de México, y el Leprocomio en Zoquiapan, Estado de México (Fig. 6).

En cuanto al Hospital para Enfermos Tuberculosos, se encontraba en la zona denominada Huipulco, inició funciones en 1935 y dependía de la Secretaría de Asistencia. Paralelamente se conformaron dispensarios antituberculosos en las ciudades de Mérida,

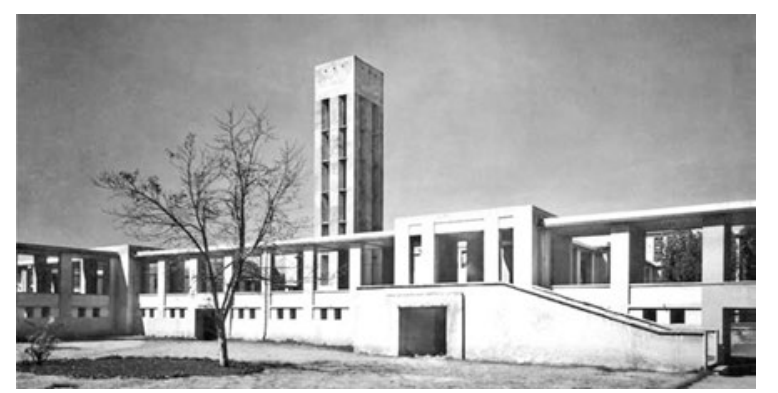

Figura 6. Hospital para enfermos tuberculosos de Huipulco (en: https://www.google.com.mx/search?q=imagen+del+sanatorio+para+enfermos+tuberculosos+de+Huipulco\&rlz=). 
Hermosillo, Tampico, Veracruz, Jalapa y Mazatlán. La creación de los dispensarios fue un reconocimiento de la importancia médico-social que se daba a la tuberculosis. Había vasos comunicantes entre lo preventivo y lo curativo.

En 1939 se inauguró el Instituto de Salubridad y Enfermedades Tropicales. Sus puntales fueron los doctores Miguel Bustamante, Manuel Martínez Báez, Gerardo Varela y Eliseo Ramírez. El Instituto constituye un hito en la historia de la clínica y la salud pública: «fue la primera institución de investigación y estudio de las enfermedades de interés para la salubridad del país» ${ }^{36}$. El Instituto, en su inicio, se ocupó de aspectos clínicos, sanitarios, docentes y de investigación referentes a paludismo, fiebre amarilla, oncocercosis, mal de pinto y parasitosis digestivas.

En 1939, la altaprevalencia de lepra dio lugar a la fundación del Leprocomio Pedro López, en Zoquiapan, Estado de México ${ }^{37}$, que se coordinaba con dispensarios antileprosos. Cabe mencionar que en esa época había horror al leproso, ser humano deformado, que se derrumbaba, y era marginado. Lo anterior dio lugar a que el establecimiento, con el tiempo, se convirtiera en un auténtico pueblo. Este Leprocomio contaba con viviendas intramuros, había talleres de carpintería, costura, herrería, una peluquería, una cocina y hasta un cementerio.

\section{Los servicios médicos para los trabajadores del riel}

En cuanto a los hospitales para los trabajadores, eran financiados por empleadores 0 trabajadores, 0 por ambos. En relación con las instalaciones para los ferrocarrileros, se constituyó una red de servicios médicos, ahora memorable y legendaria, constituida por el Hospital Colonia en el antiguo Distrito Federal, hospitales «foráneos", puestos de socorro, consultorios y vagones médicos. El Hospital Colonia dejó una huella médica contundente en México. Se trataba de una instalación modelo y única en su género, construida de acero, concreto y piedra, contaba con mobiliario y equipos modernos, se atendían problemas de medicina general y de especialidades médico-quirúrgicas, y fue uno de los primeros nosocomios verticales de México. Empezó a dar servicio el 19 de septiembre de 1936, sustituyendo a un hospital construido a base de madera. En dicho establecimiento se prestaban no solo servicios a los trabajadores del riel, sino que podían ingresar pacientes privados.

\section{Inicio de la atención médica en Petróleos Mexicanos}

Otra faceta que debe considerarse en el período cardenista respecto a hospitales para trabajadores se relaciona con la expropiación petrolera, acontecimiento ocurrido en 1938. Antes de ese año, la atención hospitalaria para los trabajadores del petróleo era insuficiente y fragmentada. Había pocos inmuebles, y algunos pertenecían a las empresas petroleras extranjeras. La mayoría de las veces, los trabajadores recibían servicios nosocomiales en organismos privados o públicos que se rentaban o subrogaban; poco después se iniciaría lo que sería un gran plan de servicios médico-hospitalarios para el personal de Petróleos Mexicanos.

\section{Hospitales lucrativos}

En este lapso de tiempo continuaron en funcionamiento hospitales privados, como la Maternidad Toriz en la ciudad de Puebla. Se fundaron otros inmuebles en todo el país, como la Clínica San Luis en la ciudad de San Luis Potosí, que abrió sus puertas en 1934, y la Clínica Neuro-psiquiátrica Dr. Manuel Falcón en el Distrito Federal, que fue inaugurada en 1937.

\section{Los «refugiados» tuvieron hospitales}

Durante los años en estudio se dio asilo político a emigrantes españoles. Eran los llamados «refugiados", quienes tuvieron problemas para recibir atención médica en los servicios para los hispanos que ya residían en el país. Los «refugiados» crearon sus propios hospitales pequeños. Los hubo en la Ciudad de México y otras ciudades del país. Los enfermos eran atendidos por médicos también «refugiados». Uno de los inmuebles más conocidos en el Distrito Federal fue la Benéfica Hispana, un esfuerzo para emparejar y hacer accesible la atención médica a todos los españoles.

Para concluir, en el sexenio cardenista hubo una renovación en la atención hospitalaria, edilicia y financiera. Asimismo, se dieron bases ideológicas sociales y públicas para la creación de los servicios médicos del Instituto Mexicano del Seguro Social. 


\title{
La medicina militar en México durante el Cardenismo (1934-1940)
}

\author{
Antonio Moreno-Guzmán \\ Escuela Médico-Militar, Ciudad de México, México
}

\section{Introducción}

La gestión presidencial del general Lázaro Cárdenas del Río (Fig. 7) fue la primera en el país con 6 años de duración, como consecuencia de su plataforma electoral apoyada en el «plan sexenal», y se caracterizó, entre otras cosas, por la ruptura con las estructuras políticas del poder previas a él y el inicio de una nueva etapa económica, política, social y educativa en el México posrevolucionario. Como parte de esa nueva visión y proyecto de país, Cárdenas fue un destacado impulsor de la modernización del Servicio de Sanidad Militar en México.

\section{Antecedentes}

Para una mejor comprensión del decidido apoyo del general Cárdenas al Servicio de Sanidad Militar, es necesario recordar que Lázaro Cárdenas ingresó desde 1913 a las filas revolucionarias con el grado de capitán segundo, y para 1928 ya había logrado alcanzar la máxima jerarquía militar, el grado de general de división ${ }^{38}$.

Importante también es destacar que durante la rebelión delahuertista, el 26 de diciembre de 1923, en las campañas en el Occidente del país en una batalla en Huejotitlán, Jalisco, el general Cárdenas fue derrotado por el general Rafael Buelna bajo las órdenes

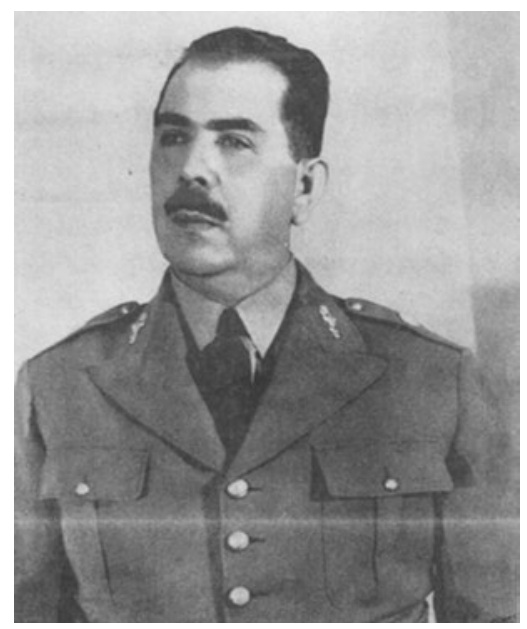

Figura 7. El general de división Lázaro Cárdenas del Río, presidente de la República (1934-1940) (en: Archivo fotográfico de la Comisión de Estudios Históricos de la Escuela Médico-Militar). del general Enrique Estrada. Ese día Cárdenas resultó gravemente herido, presentando una herida por proyectil de arma de fuego penetrante de tórax con hemotórax abundante ${ }^{39}$, y fue eficientemente atendido por el médico militar del 37. Batallón de Infantería de Aguascalientes, el mayor médico cirujano (MC) Adolfo Ochoa y Mena, egresado de la Escuela Médico-Militar en 1923, quien por cierto también resultó herido, aunque de menor gravedad, y amén de las heridas de ambos y la imposibilidad de evacuar al general Cárdenas, decidió permanecer al lado de su paciente herido a pesar de que el mismo general Cárdenas le ordenó que lo dejara, habiendo sido ambos tomados prisioneros por el general Estrada, rebelde delahuertista quien por cierto les confirió un trato muy diligente a los dos, al grado de que dio los medios y ordenó al mayor MC Ochoa que evacuara al general Cárdenas a Guadalajara, y este lo llevó al sanatorio del Dr. Carlos Barrier, quedando a partir de ese momento bajo el cuidado del Dr. Alberto Onofre. Una vez recuperados ambos de sus heridas, los liberó dándoles la opción de unirse a la rebelión o retornar a las filas gubernamentales a donde pertenecían, y tanto el general Cárdenas como el mayor M.C. Ochoa decidieron reincorporarse a las fuerzas federales ${ }^{40}$.

Finalmente, ya siendo presidente de la República, el pediatra de su hijo Cuauhtémoc fue el entonces teniente coronel MC Federico Gómez Santos ${ }^{41}$, quien fuera una autoridad en la pediatría nacional y pocos años más tarde uno de los fundadores del Hospital Infantil de México. Indudablemente, los eventos descritos con seguridad hicieron al general Cárdenas particularmente sensible a escuchar las necesidades del Cuerpo Médico Militar para mejorar las condiciones sanitarias de los soldados de México.

\section{La medicina militar durante el sexenio del general Cárdenas}

Para la toma de posesión del general Cárdenas como presidente de la República, el 1 de diciembre de 1934, el Servicio de Sanidad Militar contaba con un Hospital General Militar, un Almacén de medicinas, un Regimiento de Tren de Ambulancias, un Lazareto Militar, 15 Hospitales Militares foráneos, seis 
Enfermerías militares, 116 Secciones Sanitarias y dos Puestos de socorros ${ }^{42}$, y aunque en «números fríos" parecieran ser suficientes, la realidad era muy diferente, pues los servicios médicos del ejército, al igual que prácticamente todos los de su tipo en el país, se encontraban en muy precarias condiciones de funcionamiento. Por esa razón, las iniciativas de modernización sanitaria militar iniciadas por el general Cárdenas fueron sumamente trascendentales para la salud de los militares y sus derechohabientes.

Al inicio del sexenio cardenista, el general de brigada MC José Siurob Ramírez era el jefe del Departamento de Sanidad Militar, puesto en el que permaneció del 16 de diciembre de 1934 al 15 de junio de 1935, fecha en la que fue designado como director del Departamento Sanitario (hoy Secretaría de Salud). Fue sustituido por el general brigadier MC Andrés G. Castro, y este, a su vez, el 1 de noviembre de 1935, fue relevado por el general brigadier MC Ignacio Sánchez Neira, correligionario del general Cárdenas, quien permaneció es ese puesto por poco más de 11 años, hasta el 31 de enero de 1947. Indudablemente, la prolongada permanencia al frente de Sanidad Militar y la influencia del doctor Sánchez Neira fueron pieza clave en los proyectos de construcción tanto del Hospital Central Militar como de la Escuela Médico-Militar ${ }^{43}$ (Fig. 8).

En el año 1936, por iniciativa del mayor MC Gustavo Gómez Azcárate, se ordenó que a todos los miembros del ejército se les determinase el tipo sanguíneo, puesto que según diferentes estudios nacionales y otros realizados por él mismo se concluyó que las transfusiones sanguíneas eran un recurso terapéutico

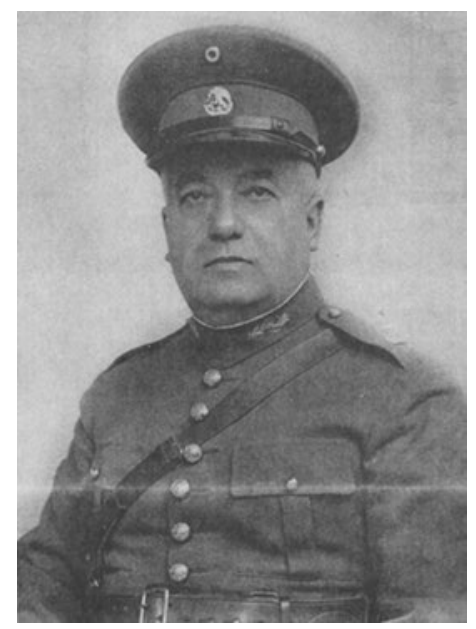

Figura 8. El general de brigada MC Ignacio Sánchez Neira, director de Sanidad Militar(1935-1947) (en: Archivo fotográfico de la Comisión de Estudios Históricos de la Escuela Médico Militar). muy importante en el tratamiento de los pacientes gravemente lesionados, medida con la que se iniciaba de manera paulatina la mejoría en el diagnóstico y el tratamiento del paciente con estado de choque hemorrágico ${ }^{44}$.

También en 1936 se dio un hecho inédito hasta ese momento y seguramente irrepetible: el 2 de mayo de ese año, el coronel MC Gustavo Baz Prada, exalumno de la Escuela Práctica Militar y de la Escuela Nacional de Medicina ${ }^{45}$, fue designado director de la Escuela Médico-Militar y simultáneamente lo fue también de la Escuela Nacional de Medicina, la cual dirigiría desde octubre de 1935 hasta el 21 de junio de 1938, cuando fue designado como rector de la Universidad Nacional Autónoma de México ${ }^{46}$, cesando como director de la Escuela Médico-Militar en agosto de 1938, poniendo de manifiesto una vez más la confianza absoluta que el general Cárdenas y el propio Consejo Universitario depositaron en los médicos militares.

Es pertinente mencionar también que, durante los aciagos años en torno a la expropiación petrolera, el embajador plenipotenciario de México ante los EE.UU. fue el general brigadier MC Francisco Castillo Nájera, desempeñándose en ese puesto de 1935 a 1945, encargándose de sortear el vendaval diplomático desatado como consecuencia de la expropiación y de la II Guerra Mundial. Importante también es mencionar que el Dr. Castillo Nájera había ocupado la presidencia de la Academia Nacional de Medicina en $1927^{47}$.

Asimismo, en ese año fueron creados en el Hospital General Militar el servicio de ortopedia, por el mayor MC Ignacio Sánchez Díaz (que ya había sido propuesto desde el año de 1932); el pabellón de cirugía plástica, por el mayor MC Joaquín de Castillo Angulo, quien además propuso que se incluyera la enseñanza de esta en las clases de la Escuela Médico-Militar; y finalmente la sala de venéreos, padecimientos que en esos años continuaban siendo una plaga entre la población militar ${ }^{48}$. Finalmente, el 10 de septiembre de 1936 se realizó la Primera Convención Nacional de Médicos Militares, siendo la sede, ni más ni menos, el Palacio de Bellas Artes recientemente inaugurado, en 1934. Contó con la presencia del presidente de la República, general Lázaro Cárdenas, y de los generales Andrés Figueroa y Manuel Ávila Camacho, secretario y subsecretario de Guerra y Marina, respectivamente. Es indudable que esto también denota la alta estima en que se tenía al Cuerpo Médico Militar en esos años ${ }^{49}$. 
Fue precisamente durante esta Convención cuando el presidente Cárdenas hizo el anuncio oficial de la construcción del futuro Hospital Central Militar, del cual él mismo había colocado la primera piedra el 13 de agosto de 1936, para lo cual creó la Comisión Organizadora y Constructora del Hospital Central Militar ${ }^{50}$ y se adquirieron los terrenos de Lomas de Sotelo, al poniente de la ciudad, donde se erigieron los edificios del Hospital Central Militar, de la Escuela Médico-Militar y de la Secretaría de la Defensa Nacional, designada así desde el 1 de septiembre de 1937, anteriormente denominada Secretaría de Guerra y Marina.

En los albores del sexenio cardenista, desde junio de 1935, fueron enviados a Berlín, Alemania, el teniente coronel MC Manuel Pérez Amador y el mayor MC Guillermo Román Carrillo para gestionar la adquisición de los equipos para el futuro Hospital Central Militar, y para 1937 se comenzaron a recibir, procedentes de Alemania, los aparatos, la maquinaria, el mobiliario, el equipo y el instrumental para el Hospital Central Militar, que aún se encontraba en construcción y que no fue inaugurado hasta el 20 de noviembre de 1942. Como dato curioso, las obras se iniciaron estando el general Cárdenas como presidente de la República y el general Ávila Camacho como secretario de Guerra y Marina ${ }^{51}$, y para cuando se concluyeron, el presidente de la República era el general Ávila Camacho y el secretario de la Defensa Nacional era el general Cárdenas, también seguramente un hecho histórico irrepetible ${ }^{52}$.

Indudablemente que el año de 1938 permanecerá indeleble en la memoria histórica mexicana como el año de la expropiación petrolera orquestada por el presidente Cárdenas, pero en particular para el Servicio de Sanidad Militar, desde luego toda proporción guardada, los sucesos más impactantes fueron el abanderamiento por vez primera de la Escuela Médico-Militar el 15 de septiembre de 1938 en el Estadio Nacional, a manos del secretario de la Defensa $\mathrm{Na}-$ cional general Ávila Camacho, y muy particularmente el nacimiento de la Escuela para Enfermeras del Ejército, cuya creación fue decretada por Lázaro Cárdenas el 21 de marzo de 1938, aunque cabe aclarar que desde 1894 el coronel MC Fernando López y Sánchez Román, a la sazón director del Hospital Militar de Instrucción, dio inicio al curso de formación para enfermeras militares en el interior del hospital militar, y para cuando fue designado el primer director del Hospital General de México, en 1905, abrió de inmediato el curso para enfermeras civiles en ese nosocomio. Aunque desde las postrimerías del siglo XIX se contó con mujeres enfermeras en el ejército, no fue sino hasta que el presidente Cárdenas decretó la creación de la Escuela para Enfermeras de Ejército cuando realmente se inició la formación académica especializada y específica de las enfermeras militares mexicanas.

Esta Escuela fue ubicada en el interior del Hospital General Militar en 1938, siendo su primer director el coronel MC Donato Moreno Muro ${ }^{53}$, y para 1942 ocupó las alas norte y sur del sexto piso de la primera sección del entonces recién inaugurado Hospital Central Militar. La Escuela, años más tarde, en 1946, cambió su nombre a Escuela Militar de Enfermeras y Parteras, para finalmente llegar a la actual denominación de Escuela Militar de Enfermeras a partir de 1963. En sus inicios todas las alumnas eran externas y paulatinamente fueron asimilándose, hasta que en 1952 todas las cadetes fueron incluidas en el sistema de internado permanente durante todos sus estudios.

En cuanto a su jerarquía militar, aunque desde 1947 egresaban con el grado de subteniente enfermera, esto no quedó oficialmente asentado hasta el decreto del presidente Ruiz Cortines del 5 de enero de 1957, en el cual también se especificaba que podían ascender en la escala jerárquica, mediante riguroso examen de promoción, hasta el grado de capitán primero enfermera; esta situación ha ido cambiando con los años y actualmente alcanzan en el activo el grado de coronel enfermera.

Finalmente, esta Escuela se trasladó del Hospital Central Militar a su propio edificio, ubicado a espaldas del Hospital Militar, en el año de 1964, y desde 2011 sus alumnas egresan con el grado militar de subtenientes enfermeras y el grado académico de licenciadas en enfermería ${ }^{54}$.

En cuanto a los varones dedicados a la enfermería militar, debo mencionar lo siguiente: como antecedente de la Escuela Militar de Oficiales de Sanidad, es indispensable señalar que, desde el 21 de febrero de 1927, el mayor MC Juan Gil Saldaña Alcocer inició, aunque de manera irregular, una escuela para el adiestramiento de enfermeros varones para el servicio del ejército. Esta nació por la inquietud del mayor MC Saldaña de crear una escuela para formar Enfermeros y Camilleros Militares que fueran capaces de auxiliar al médico militar en sus actividades técnicas, tácticas y administrativas, y principalmente para proveer los primeros auxilios a los heridos en combate. Por ello se iniciaron cursos de manera irregular en el Hospital Militar de Instrucción, pero no fue hasta el año de 1937 cuando el mayor MC Saldaña Alcocer vio cristalizado su proyecto, al haber sido autorizado el primer curso 
formal con plan de estudios, aunque sin presupuesto para ello. Ingresaron los primeros 37 alumnos con un perfil de ingreso que incluía, entre otros aspectos, contar con estudios incompletos de enfermería o medicina, y vocación por la carrera de la salud y la atención de heridos ${ }^{55}$. No fue sino hasta el 23 de octubre de 1939 cuando, por decreto presidencial de Lázaro Cárdenas, fue oficialmente creada la Escuela de Enfermeros Militares, habiendo sido ubicada dentro del Hospital General Militar, sin presupuesto y con alumnos externos. Años más tarde, por decreto del presidente Manuel Ávila Camacho, del 15 de abril de 1943, la Escuela se cambió el 1 de enero de 1944 al Hospital Central Militar y se inauguró oficialmente el 17 de enero de 1944, abanderada por el secretario de la Defensa Nacional hasta el 30 de abril de 1947 en la explanada del Hospital Central Militar ${ }^{56}$.

El 19 de julio de 1954 cambió su nombre de Escuela de Enfermeros Militares por el de Escuela de Oficiales de Sanidad Militar, por lo dispuesto en el decreto presidencial del 5 de julio de 1954. El 9 de noviembre de 1970 fue dotada de un edificio propio y finalmente volvió a cambiar su nombre el 24 de febrero de 1980 por el actual de Escuela Militar de Oficiales de Sanidad. Sus alumnos egresan, desde 2011, con el grado militar de subteniente de sanidad y el grado académico de licenciado en salud pública y urgencias médicas ${ }^{57}$.

\section{Consideraciones}

El impulso sin precedente que recibió el Servicio de Sanidad Militar por parte del presidente Lázaro Cárdenas fue determinante para que la medicina militar mexicana alcanzara su pleno desarrollo en la segunda mitad del siglo XX, al cambiar para siempre el paisaje urbano al poniente de la Ciudad de México con la construcción del nuevo Hospital Central Militar, iniciada por el general Lázaro Cárdenas en 1936 y culminada por el general Manuel Ávila Camacho en 1942, que al momento de su inauguración fue el primer hospital vertical de la nación y el más grande y moderno de toda América Latina, con una capacidad instalada de 1000 camas y con un sistema de atención para los miembros del ejército y sus familiares que se tomó como modelo y pronto se vio replicado en las nacientes instituciones de seguridad social de nuestro país ${ }^{58}$.

Además, el general Cárdenas completó el proyecto de construcción de Hospital Central Militar con la creación de las escuelas de enfermería para mujeres y varones, la Escuela Militar para Enfermeras del Ejército en 1938 y la Escuela de Enfermeros Militares en 1939, con las que indudablemente robusteció la calidad de la atención que recibieron a partir de entonces los militares y sus derechohabientes. 


\title{
Conclusiones finales del simposio
}

\author{
Martha Eugenia Rodríguez \\ Departamento de Historia y Filosofía de la Medicina, Facultad de Medicina, Universidad Nacional Autónoma de México, Ciudad de México, México
}

El México gobernado por el General Lázaro Cárdenas del Río era complejo. Se caracterizó por carencias, insalubridad, desnutrición (fundamentalmente la infantil) y padecimientos de la pobreza, como los infectocontagiosos, entre ellos el tifo y la lepra, que podían ser prevenidos, en gran medida, por medio de la educación. Para combatir la situación médico-sanitarista, el gobierno sexenal emprendió importantes políticas que modificaron las estadísticas de morbimortalidad del país. En 1934, la expectativa de vida al nacer era de 33 años, y en 1940 fue de 41.5 años. La tasa de defunciones pasó de 26.6 a 22.8 por 1000 habitantes. Los resultados se debieron al interés gubernamental, que incrementó el presupuesto para la salud. Asimismo, se debieron al trabajo institucional, el realizado por el Departamento de Salubridad Pública, pero también el de otros organismos, entre ellos los Servicios Coordinados que llegarían a zonas rurales, el Instituto de Higiene, la Escuela de Salubridad, el Instituto de Salubridad y Enfermedades Tropicales, la Secretaría de Asistencia Pública y los diversos hospitales generales y especializados, civiles y militares.

Las campañas contra las enfermedades desempeñaron un papel importante, fundamentalmente para las causadas por microbios, virus, parásitos y vectores. Fueron campañas bien implementadas que contemplaban la asistencia al enfermo, educación en materia de salud, hábitos higiénicos, ingeniería sanitaria, etcétera. En ellas participaron no solo médicos, sino también enfermeras visitadoras, ingenieros y técnicos.

Por su parte, la red hospitalaria fue amplia. Se conformaron hospitales públicos de vanguardia, entre ellos el Sanatorio de Tuberculosis y el Leprocomio en Zoquiapan, Estado de México. También se dio cobertura a los ferrocarrileros, a los empleados de petróleos y a los refugiados españoles, y se inició durante el Cardenismo la construcción del nuevo Hospital Central Militar. El Servicio de Sanidad Militar fue objeto de un impulso sin precedente; además de empezar la edificación del citado hospital, se crearon escuelas de enfermería para mujeres y hombres. En suma, se puede afirmar que durante el Cardenismo el balance en materia de salud fue positivo. Empezó un control por las enfermedades infectocontagiosas que puso las bases para futuros trabajos, al grado de que hoy en día preocupan más las enfermedades crónicas.

\section{Bibliografía}

1. González Marín S, Bosque Lastra M. Logros del cardenismo. México: UNAM, Instituto de Investigaciones Bibliográficas, Biblioteca y Hemeroteca Nacionales; 1996. p. 27.

2. Aguilar Rivera JA. Los gobiernos posrevolucionarios, 1921-1945. En: Florescano E, coordinador. Arma la historia. La nación mexicana a través de dos siglos. México: Grijalbo; 2009. p. 187.

3. INEGI. Población. Disponible en: http://www.beta.inegi.org.mx/temas/ estructura

4. Aguilar Rivera JA. Los gobiernos posrevolucionarios, 1921-1945. En: Florescano E, coordinador. Arma la historia. La nación mexicana a través de dos siglos. México: Grijalbo; 2009. p. 188.

5. González Marín S, Bosque Lastra M. Logros del cardenismo. México: UNAM, Instituto de Investigaciones Bibliográficas, Biblioteca y Hemeroteca Nacionales; 1996. p. 42.

6. Cárdenas C. Cárdenas por Cárdenas. México: Penguin Random House Grupo Editorial; 2016. p. 404.

7. Campos Navarro R. La antropología médica hace su primera aparición en México en la Escuela Superior de Medicina Rural del IPN. En: Medicina mexicana, dos siglos de historia, 1810-2010. Viesca C, coordinador. México: Bayer de México; 2011. p. 317.

8. Baz G. Palabras de Gustavo Baz en septiembre de 1936 al enviar a los primeros 260 estudiantes al campo mexicano en servicio social. En: Conmemoración del cincuenta aniversario de la instauración del servicio social en México. México: Secretaría de Salud, 1986, p.19. Agostoni C, Ríos Molina A. Las estadísticas de salud en México. Ideas, actores e instituciones, 1810-2010. México: UNAM y Secretaría de Salud; 2010. p.179-80.

9. Álvarez Amézquita J,et al. Historia de la salubridad y de la asistencia en México. México: Secretaría de Salubridad y Asistencia; 1960, II: 382.

10. Aguilar Rivera JA. Los gobiernos posrevolucionarios, 1921-1945. En: Florescano E, coordinador. Arma la historia. La nación mexicana a través de dos siglos. México: Grijalbo; 2009. p. 190.

11. Souza MI. Isaac Costero: vivir para la ciencia. México: Academia Nacional de Medicina, CONACYT, Intersistemas S.A. de C.V.; 2014. p. XVII.

12. Carrillo AM. Salud pública y poder en México durante el Cardenismo, 1934-1940. En: DYNAMIS. Acta Hisp Med Sci Hist Illus. 2005;25:145-78.

13. Archivo Histórico de la Secretaría de Salud (AHSSA). Fondo: Salubridad Pública. Sección: Presidencia. Serie: Acuerdos presidenciales. C. 47, exp. 29, f. 57.

14. AHSSA, F: CSG, Actas, c. 1, exp. 1, p. 202

15. Prida M. La enfermera en la campaña contra las enfermedades transmisibles. En: Memoria de la Cuarta Asamblea Nacional de Cirujanos. México; 1940. p. 275-8.

16. AHSSA, F: Salubridad Pública, S: Presidencia, Se: Acuerdos presidenciales, L. 1, exp. 1, f. 41.

17. Álvarez Amézquita J,et al.Historia de la salubridad y de la asistencia en México. México: Secretaría de Salubridad y Asistencia; 1960, II: 439.

18. Aboites Aguilar L. El último tramo, 1929-2000. En: Nueva historia mínima de México. 5. ${ }^{\text {a }}$ reimp. México: El Colegio de México; 2008. p. 262-302.

19. Cárdenas L. Discurso al protestar como presidente de la República ante el Congreso de la Unión el 30 de noviembre de 1934. En: Los presidentes de México ante la nación. 1821-1966. 4 vols. México: CXLVI Legislatura, Cámara de Diputados; 1966. IV: 11.

20. Cárdenas L. Informe de Gobierno, $1^{\circ}$ de septiembre de 1935, En: Los presidentes de México ante la nación. 1821-1966. 4 vols. México: CXLVI Legislatura, Cámara de Diputados; 1966. IV: 34-35.

21. Cárdenas L. Informe de gobierno, $1^{\circ}$ de septiembre de 1936. En: Los presidentes de México ante la nación. 1821-1966. 4 vols. México: CXLVI Legislatura, Cámara de Diputados; 1966. IV: 59-60.

22. Alba F. Crecimiento demográfico y transformación económica, 19301970. En: vv.aa. El poblamiento de México. IV. México en el siglo XX. México: Secretaría de Gobernación/CONAPO; 1993. p.77-80.

23. Benítez Zenteno R. La expansión demográfica de México, 1895-1970. En: Dinámica de la población de México. México: El Colegio de México; 1970. p. 5-11. 
24 Bustamante ME. Gac Med Mex. 1934;LXV:181-4. Reproducido en: Agostoni C, Ríos Molina A. Las estadísticas de salud en México. Ideas, actores e instituciones, 1810-2010. México: UNAM/Secretaría de Salud; 2010.p.207-20.

25. Angelini A. La higiene y la medicina sociales. Gac Med Mex. 1933;LXIV:122-36.

26. Alonso Concheiro A. Cronología médica mexicana. Cinco siglos. México: Consejo de Salubridad General/Academia Nacional de Medicina/Academia Mexicana de Cirugía/Siglo Veintiuno editores; 2010. p.202.

27. Bustamante $M$, Viesca $C$, Villaseñor $F$, et al. La salud pública en México, 1959-1982. México: SSA; 1982

28. Bustamante M. Observaciones sobre la mortalidad general en México, de 1922 a 1969. En: Almada Bay I, compilador. La mortalidad en México. 1922-1975. México: IMSS; 1982. p. 45-56.

29. Álvarez Amézquita J, Bustamante ME, López Picazos A, Fernández de Castillo F. Historia de la salubridad y de la asistencia en México. 4 vols. México: Secretaría de Salubridad y Asistencia; 1960. Vol. III.

30. Celis A, Nava J. Patología de la pobreza. En: Almada Bay I, compilador La mortalidad en México. 1922-1975. México: IMSS; 1982. p. 297-314.

31. Valdespino G, JL, Río Z, A del, Escobar G, A, Mora G, JL, editores. Una Institución Académica Mexicana y dieciséis investigadores distinguidos. Quincuagésimo aniversario del INDRE. México: INDRE, Secretaría de Salud; 1994

32. Viesca C, Díaz de Kuri M. El Hospital Infantil de México. 50 años de Historia. México: Hospital Infantil; 1993.

33. Meyer J. México entre 1934 y 1998. En: Historia de México. Van Wobeser G, coordinadora. México: Academia Mexicana de la Historia, Fondo de Cultura Económica; 2010. p. 249.

34. Archivo Histórico de la Secretaría de Salubridad y Asistencia. Fondo Salubridad Pública. Sección 5, Caja 5, Expediente 10.

35. Villalbazo Reyes JA, Morales Meneses J, elaboradores; Alonso Gutiérrez JF, coordinador. Guía del Fondo Beneficencia Pública. Addenda. México: Secretaría de Salud, Oficialía Mayor: 1990. p. V, VI.

36. Martínez Barbosa X, Martínez Cortes F, Rivero Serrano O. El Consejo de Salubridad General (1925-2000). México: Smith Kline Beecham; 2000. p. 45.

37. Chávez I. México en la cultura médica. México: Biblioteca de la Salud Instituto de Salud Pública, Fondo de Cultura Económica; 1987. p. 143.

38. Poblett-Miranda M. Lázaro Cárdenas. En: Villapando JM, director. Grandes protagonistas de la historia mexicana. México: Planeta De Agostini; 2002.p.153.

39. Krauze E. Lázaro Cárdenas, general misionero. $1^{\mathrm{a}}$ edición, $5^{\mathrm{a}}$ reimpresión. México: Fondo de Cultura Económica;2002. p. 24.

40. Lozoya Solís J. La Escuela Médico Militar de México. México: Edición del autor;1977. p.81.
41. Lozoya Solís J. La Escuela Médico Militar de México. México: Edición del autor;1977. p.271.

42. Gutiérrez SJL. Historia del Servicio de Sanidad Militar en México. Tomo III. Ciudad de México: Estado Mayor de la Secretaría de la Defensa Nacional; 1987. p. 186.

43. Gutiérrez SJL. Historia del Servicio de Sanidad Militar en México. Tomo III. Ciudad de México: Estado Mayor de la Secretaría de la Defensa Nacional; 1987. p. 190.

44. Gutiérrez SJL. Historia del Servicio de Sanidad Militar en México. Tomo III. Ciudad de México: Estado Mayor de la Secretaría de la Defensa Nacional; 1987. p. 193.

45. García-Procel E, coordinador. Academia Nacional de Medicina de México. Presidentes 1864-2006. p.61.

46. Gustavo Baz Prada.(Consultado el 12 de agosto de 2016.)Disponible en: http://bibliohistorico.juridicas.unam.mx/libros/1/254/100.pdf

47. García-Procel E, coordinador. Academia Nacional de Medicina de México. Presidentes 1864-2006. p.53.

48. Gutiérrez SJL. Historia del Servicio de Sanidad Militar en México. Tomo III. Ciudad de México: Estado Mayor de la Secretaría de la Defensa Nacional; 1987. p. 197.

49. Gutiérrez SJL. Historia del Servicio de Sanidad Militar en México. Tomo III. Ciudad de México: Estado Mayor de la Secretaría de la Defensa Nacional; 1987. p. 195.

50. Moreno-Guzmán A. Historia de la cirugía general en el Hospital Central Militar. Rev Sanid Milit Mex 2013;67:128-35

51. Ruiz-Massieu A, Flores ME. Gabinetes presidenciales. México: Costa-Amic; 1988.p.192.

52. Moreno-Guzmán A. La formación de los cirujanos militares mexicanos, 1947-1970. México: Edición del autor; 2016. p.67.

53. Calva CE. Donato Moreno Muro. Coronel Médico Cirujano. Rev Sanid Milit Mex.2006;60:429-35.

54. Escuela Militar de Enfermeras. Cuna de mujeres leales. 75 aniversario. México: Secretaría de la Defensa Nacional, Dirección General de Comunicación Social; 2015.p. 62.

55. Moreno-Guzmán A. El Cuerpo Médico Militar y el Servicio Sanitario durante la Revolución Mexicana. Rev Sanid Milit Mex. 2014;68:325-40.

56. Gutiérrez SJL. Historia del Servicio de Sanidad Militar en México. Tomo III. Ciudad de México: Estado Mayor de la Secretaría de la Defensa Nacional; 1987. p. 208.

57. Centro Militar de Ciencias de la Salud. México: Secretaría de la Defensa Nacional, Dirección General de Comunicación Social, Sección de Difusión Interna; 2016. p. 45.

58. Sánchez NI. El Hospital Central Militar. Gral.de Bgda. M. C. Ignacio Sánchez Neira. Director de Sanidad Militar. México: Edición del autor; 1943. 